\title{
The comparative incidence of malignant disease in rheumatoid arthritics exposed to different treatment regimens
}

\author{
B R I A N L A ZLEMAN \\ From the Department of Rheumatology, Addenbrooke's Hospital, Hills Road, Cambridge \\ PRESENTED BY M DE SILVA \\ Senior Registrar, Addenbrooke's Hospital, Cambridge
}

SUMmary Cytotoxic drugs are effective in disease suppression and affect the immune system. The correlation between these is poor, in many patients, at the dosage used in man. This may partly explain why the large increased risk of neoplasia, initially predicted, has not been shown to follow the use of cytotoxic therapy.

The cause of death in patients with rheumatoid arthritis has been assessed both by surveys based on clinical populations and by reviews of necropsy records. There have been few attempts to assess the influence of drug therapy on mortality, or on the incidence of neoplasia. In addition, evidence is accumulating to suggest that lymphoreticular tumours may be associated with autoimmune disease.

There have now been several long-term studies of patients with rheumatoid arthritis treated with immunosuppressives; azathioprine and cyclophosphamide in particular. These studies are reviewed and it is concluded that the incidence of most of the common cancers is not increased.

The use of immunosuppressive drugs in rheumatoid arthritis has, in general, proved to be of benefit. ${ }^{82} 143$ Azathioprine or cyclophosphamide, started early in the course of rheumatoid arthritis, have been shown to be equally effective and similar in their efficacy to gold therapy. ${ }^{38}$ Since a short course of such treatment does not produce a lasting remission, long-term administration would be expected in many instances. This focuses attention on the adverse effects of prolonged administration of these drugs including bone marrow suppression, susceptibility to infection and the potential development of malignant tumours. Some of the potential toxic side effects are listed in table 1 .
TABLE 1 Toxicity of immunosuppressive drugs

\begin{tabular}{lllll}
\hline & $\begin{array}{l}\text { Cyclophos- } \\
\text { phamide }\end{array}$ & Azathioprine & $\begin{array}{l}\text { Chloram } \\
\text { bucil }\end{array}$ & Methotrexate \\
\hline $\begin{array}{l}\text { Gastrointestinal } \\
\text { intolerance }\end{array}$ & + & & & \\
Infection & + & + & + & + \\
Cystitis & ++ & + & + & + \\
Hair loss & ++ & - & - & - \\
Hepatic damage & - & - & + & + \\
Leucopenia & ++ & + & - & ++ \\
Azoospermia & ++ & + & ++ & + \\
Anovulation & ++ & - & ++ & + \\
Teratogenesis & + & - & ++ & - \\
Neoplasia & + & $+?$ & + & + \\
\hline
\end{tabular}

The relative contributions to the patient's increased susceptibility to infections arising from the disease itself, and from the frequent concomitant steroid therapy, remain difficult to evaluate. Infections with organisms such as cytomegalovirus, herpes simplex, mycobacterium tuberculosis and fungi are possible. The use of cyclophosphamide may be accompanied by alopecia, haemorrhagic cystitis and bladder fibrosis; it may also lead to ovarian failure and azoospermia. Azathioprine is apparently free of such side effects.

Cytostatic drugs are potentially teratogenic in man, although several women receiving azathioprine have conceived and delivered healthy babies. Methotrexate, if given repeatedly, can lead to hepatic fibrosis. The most obvious effect of these drugs is on the haemopoietic system. Since they affect rapidly proliferating cells they have an effect on bone marrow precursors, such as stem cells and the myeloid and erythroid, as well as the lymphoid, series.

\section{Induction of malignant disorders}

The most difficult general problem to assess, associated with the use of immunosuppressive agents, is the potential threat of the development of neoplasia. It is 
theoretically possible that the development of a neoplasm may occur many years after continuous immunosuppressive therapy or even after discontinuation of such therapy. It took many years to recognise the increased risk of leukaemia that occurs in patients who had received radiation therapy for ankylosing spondylitis some 20 to 25 years previously. ${ }^{37}$

Over the last decade considerable evidence has accumulated suggesting that immunosuppressive and cytotoxic drugs cause an increased incidence of malignancy, particularly when used for long periods. Most of the evidence is derived from studies of patients receiving organ transplants, and more anecdotal evidence comes from the use of these drugs in rheumatic diseases. Animal experiments show that immunosuppression facilitates the transplantation of malignant cells, increases the incidence of virus-induced cancers and accelerates the growth of metastases. Perhaps the expected increased risk of neoplasia, initially predicted, has not materialised because there is little correlation between efficacy of disease suppression and effect on the immune system in man.

Recently a prospective study ${ }^{115}$ of 3823 patients who had received a renal transplant and of 1349 other patients, including cases of rheumatoid arthritis, receiving cytotoxic drugs (particularly azathioprine), showed no clear evidence that immunosuppressive drugs produce an increased risk of most of the common cancers. However, few of the patients had been under observation for over five years, and an increase may appear later. Follow-up of the renal transplant recipients showed an almost 60 -fold increase of non-Hodgkin's lymphoma together with an excess of squamous cell skin cancer and mesenchymal tumours. The patients without transplants showed an excess of the same tumours, though to a lesser extent.

The most striking excess of lymphoid tumours occurred as non-Hodgkin's lymphomas (mainly described as reticulum cell sarcomas and microgliomas) in the transplant series, which confirms what has been reported before. ${ }^{94}$ This excess of lymphoid tumours was extraordinary in its magnitude, predilection for the brain and very short induction period. Possible hypotheses to explain these findings include disturbance of immunosurveillance or of graft-versus-host reaction, chronic antigenic stimulation and the effect on oncogenic viruses. For each of these there is laboratory support. The short induction period, sometimes within a few months after transplantation, suggests a viral origin, since transformation could take place immediately if the virus was already present.

That patients without transplants also showed an excess of lymphoid tumours suggests that immunosuppression is important, though the presence of a graft may contribute to tumour development, since in transplant recipients the excess was much greater. Other observations support the primary importance of immunosuppression. Firstly, there was no tendency for lymphoid tumours to be more common in recipients who experienced repeated rejection crises, nor in those who had had multiple grafts. Secondly, a study of patients treated by renal dialysis, who have severely depressed immune reactions, showed that they also had an increased incidence of non-Hodgkin's lymphoma, and that in one of them, who had never been given immunosuppressive drugs, the lymphoma affected the brain. Thirdly, certain rare hereditary disorders characterised by major immunological impairment, such as the Wiskott-Aldrich syndrome, are associated with an increased risk of lymphomas.

A direct mutagenic effect of the chemical agents appears to be unlikely because of the abrupt increase in incidence, the subsequent constancy of the risk from the time of first exposure and the lack of any noticeable dose effect, all of which are in pronounced contrast to the usual findings with known chemical carcinogens.

Only four patients without transplants developed non-Hodgkin's lymphoma (three of whom were treated with azathioprine). ${ }^{115} \mathrm{~A}$ three-fold excess of lymphomas was observed in a large series of patients with rheumatoid arthritis in Finland ${ }^{101}$ and it was not clear whether this was associated with any particular form of treatment. However, cases of lymphoma affecting the brain in patients without transplants, but receiving immunosuppressive drugs, have been reported. ${ }^{127} 220221$

The risk of non-Hodgkin's lymphoma in the study of Kinlen et $a l^{115}$ was smaller in patients without transplants than in transplant recipients. Differences in the type, amount and duration of drug treatment were, however, substantial. Azathioprine was given initially only to $64 \%$ of patients in the non-transplant series and was continued for over two years in less than $30 \%$, whereas azathioprine was continued in over $90 \%$ of the transplant recipients throughout the period of observation. Similar differences were observed in the case of prednisone.

Walder et al in $1971^{231}$ emphasised the common occurrence of squamous carcinomata in transplant recipients. Kinlen et $a l^{115}$ also reported a ten-fold increase in mortality from skin cancer in transplant recipients. In the latter study there were three cases of acute leukaemia, all in transplant recipients, compared with the expected figure of less than one, and six mesenchymal tumours when none was expected; three of these were in the non-transplant group. There were four cases of bladder cancer in patients 
without transplants and the risk of this disease is related specifically to the use of cyclophosphamide.

In order to try to distinguish between the effects of immunosuppressive therapy and the disease process in rheumatoid arthritis, surveys of the cause of death must be examined.

\section{Cause of death}

The cause of death in patients with rheumatoid arthritis has been assessed both by surveys based on clinic populations and by reviews of necropsy records. ${ }^{1335575102151156167219226}$ There have been few attempts to assess the influence of drug therapy on mortality or on the incidence of neoplasia.

Most surveys of the causes of death in rheumatoid arthritis agree that patients with the disease have a higher mortality rate than the rest of the population, though they die not so much from specific complications of the disease as from all the usual causes of death, but at an earlier age than usual.

In 1970 Uddin et $a^{219}$ reviewed 475 patients with rheumatoid arthritis, first seen between 1954 and 1966. The cumulative survival rate was determined in each year for up to ten years, compared with that of a normal matched population. This was lower than expected for each sex at each year of follow-up, particularly in the later years.

Cobb et al in $1953^{33}$ followed 583 patients for a mean duration of $9 \frac{1}{2}$ years. The overall mortality rate was 24.4 per 1000 patients per year in contrast to 18.9 for a comparable group matched for age and sex. More recently, Isomaki et al in $1975^{102}$ observed 1000 patients with rheumatoid arthritis for three years. All were over 40 years of age. Deaths were compared with an age and sex matched control population. The 122 deaths in the rheumatoid group compared with 69 in the controls. Deaths from cancer were fewer in the rheumatoid group.

It is difficult to compare studies of the causes of death in rheumatoid patients. Most studies rely on certified causes of death which, on occasion, do not correlate well with findings at necropsy. There are also differences in the periods of follow-up.

The improved hospital supervision of rheumatoid patients and the use of an expanded range of therapeutic agents in the last decade may invalidate comparisons with early studies.

Reports of the incidence of neoplasia, independent of therapy, vary between studies. Only Moesman in $1969^{151}$ found a greater frequency of malignant disease in a small group of elderly patients.

\section{Induction of malignant disorders}

In view of reports of malignancy, Hunter et al in
$1975^{95}$ carried out chromosomal studies in patients receiving long-term azathioprine. This showed a two-fold increase in chromosomal abnormalities in patients, similar to that reported during cyclophosphamide treatment of rheumatoid arthritis by Tolchin et al in $1974 .{ }^{216}$ Since similar changes may occur with phenylbutazone, and a variety of other drugs, its significance will have to await long-term observation.

Evidence is accumulating to suggest that lymphoreticular tumours may be associated with autoimmune disease. ${ }^{62149211}$ Patients with Sjögren's syndrome are especially prone to develop extensive lymphocyte infiltrates in extrasalivary organs with pseudolymphoma formation and occasional progression to malignant lymphoma. ${ }^{6}$ Three series of patients with systemic lupus erythematosus have shown an increased risk of cancer. ${ }^{29} 52126$

Azathioprine has been widely used as an immunosuppressive agent in the United Kingdom, whereas cyclophosphamide is used more widely in North America.

Owing to the influence of de Seze and Kahn, ${ }^{46}$ chlorambucil has been the most widely used immunosuppressive drug in France. This choice was based on the use of the drug in chronic lymphatic leukaemia where there were hopes that it would have a greater affinity for lymphocytes. The enthusiasm for the drug declined during the 1970's on account of its side effects. Deshayes $e t$ al $^{47}$ summarised the results concerning side effects of chlorambucil noted by several French authors in a total of 495 patients, and compared these to the effects of azathioprine and cyclophosphamide.

Treatment linked mortality probably depends on the duration of exposure to risk, which is difficult to determine for a treatment with prolonged aftereffects. Its assessment depends on the length of time for which the patients are followed after treatment has begun, and on whether all deaths are included or only those which can be attributed to the treatment. Most important is the fact that no true control group exists, and a comparison can only be made with the life expectancy of the general population of the same age or that of a random group of rheumatoid patients.

Farber et $\mathrm{l}^{64}$ compared 126 patients treated with cytotoxic drugs with 126 age and sex matched rheumatoid arthritic patients in hospital during the same years but who did not receive cytotoxic therapy. Patients predominantly received nitrogen mustard or cyclophosphamide or both. The patients were followed from 1965 to 1974 and no increase in malignancy was recorded, nor were any lymphomas seen. This view is not shared by others. Parsons and co-workers in $1974^{169}$ carried out a long-term follow-up of patients with rheumatoid arthritis treated with cyclophosphamide. They found eight 
malignancies including seven of the lymphoproliferative variety, but the incidence of Sjögren's syndrome is not recorded. Pollock et al in $1973^{179}$ reported two malignancies in rheumatoid arthritic patients treated with cyclophosphamide (both solid tumours), and a recurrence of a previously treated melanoma was reported in a patient following cyclophosphamide therapy. ${ }^{138}$

Renier et al in $1978^{1 \times 4}$ followed 131 cases of rheumatoid arthritis for at least four years and recorded 13 deaths through malignant disease, of which seven were haemopoietic neoplasms. Cancer was diagnosed in five other patients. Eighteen of the 131 patients had received chlorambucil, and then later cyclophosphamide, and seven of the cases of malignant disorder were observed in these 18 patients.

Four cases of leukaemia have arisen in association with rheumatoid arthritis following treatment with azathioprine,,$^{32131196}$ and in two cases following treatment with cyclophosphamide. ${ }^{131}$

Kahn et al in 1979, ${ }^{108}$ using results from a collective retrospective study, calculated the incidence of acute leukaemia during and after cytotoxic therapy. The results of this study, which included 2006 patients whose average age was 55 years, are shown in table 2 . A total of 1711 patients had received chlorambucil and were followed for one to 13 years. One third of the patients were not followed up. Kahn et al ${ }^{108} \mathrm{com}$ pared these figures with cyclophosphamide treatment (three cases of leukaemia in a co-operative study of 229 patients) and with azathioprine, where Seidenfeld et al $^{196}$ noted an incidence of $1 \cdot 3 \%$. Kahn et al emphasised that no case of leukaemia was seen in patients who received less than $1 \mathrm{~g}$ chlorambucil and in whom the duration of treatment was less than six months. The nature of the acute leukaemia with chlorambucil was variable, and the delay from the beginning of treatment to the appearance of leukaemia was $5 \cdot 7 \pm 2.9$ years. $^{108} 145$ The risk of developing leukaemia seems more pronounced in children suffering from chronic rheumatoid arthritis; three cases out of 40 infants treated with chlorambucil compared with 0 out of 160 control infants. ${ }^{23}$

TABLE 2 Results of a survey of the incidence of acute leukaemia during and after therapy with chlorambucil (Kahn et $\left.a l^{108}\right)$

\begin{tabular}{lcc}
\hline Reason for therapy & Number in study & $\begin{array}{l}\text { Number of cases } \\
\text { of leukaemia }\end{array}$ \\
\hline Rheumatoid arthritis & 1612 & 12 \\
Systemic lupus erythematosus & 44 & 0 \\
Psoriatic arthritis & 35 & 4 \\
Others & 20 & 0 \\
Total & 1711 & 16 \\
\hline
\end{tabular}

\section{Study of cause of death with particular reference to azathioprine}

In view of the few attempts to assess the influence of drug therapy on the incidence of neoplasia, the author initiated a survey on a group of closely followed patients with rheumatoid arthritis predominantly treated with azathioprine. ${ }^{125}$ The patients in this study were unusual in that treatment with azathioprine was started, in some patients, as long as 11 years previously, often in high dosage (5 $\mathrm{mg} / \mathrm{kg} /$ day) and sodium aurothiomalate (Myocrisin) had been given in combination. For purposes of comparison, an analysis of mortality was performed by calculation of expected mortality in the patient group, based on rates for a similar age and sex distributed sample of the general population.

A total of 311 patients with classical or definite rheumatoid arthritis was studied over this 11-year period. Follow-up was complete in all patients. The death certificate was obtained for all the 46 who died, and results of necropsy were available in 19 cases.

A total of 214 patients received cytotoxic therapy (table 3 ) and 63 received cyclophosphamide (range $0 \cdot 2$ to 8 years) or chlorambucil, alone or in combination with azathioprine, at some stage in their disease. The mean duration of treatment in the 151 patients treated with azathioprine alone was $2 \cdot 5$ (range 1 to 11) years, a total of 500 patient years.

The tumours diagnosed in both the dead and the survivors are recorded in table 4 . Five patients have had apparently successful treatment of their neoplasm (three patients had received cytotoxic therapy before the discovery of the neoplasia). Ten of the 20 neoplasms occurred in patients who were receiving cytotoxic therapy (table 5). There is no evidence to link rheumatoid arthritis with any particular form of tumour.

The one case of lymphosarcoma occurred in a 71-year-old patient, without evidence of Sjögren's syndrome, who had not received cytotoxic therapy.

TABLE 3 Malignancy associated with treatment

\begin{tabular}{|c|c|c|c|c|}
\hline Method of management & & $\begin{array}{l}\text { Number of } \\
\text { patients }\end{array}$ & $\begin{array}{l}\text { Number of } \\
\text { malignancies }\end{array}$ & $\begin{array}{l}\text { Incidence } \\
(1 / 1)\end{array}$ \\
\hline Total group & & 311 & 20 & $<6$ \\
\hline $\begin{array}{l}\text { Not receiving cytoxic } \\
\text { agents }\end{array}$ & & 97 & 10 & 10 \\
\hline Receiving cytoxic agents & & 214 & 10 & 5 \\
\hline $\begin{array}{l}\text { Azathioprine alone } \\
\text { Azathioprine + either } \\
\text { chlorambucil or }\end{array}$ & 151 & & 6 & 4 \\
\hline $\begin{array}{l}\text { cyclophosphamide } \\
\text { Either chlorambucil } \\
\text { or cyclophosphamide }\end{array}$ & $\begin{array}{l}52 \\
11\end{array}$ & -214 & 2 & - \\
\hline
\end{tabular}


TABLE 4 Neoplasia occurring in 311 patients with rheumatoid arthritis (1966-1976)

\begin{tabular}{lc}
\hline Type of neoplasm & \\
\hline Lung/bronchus & 5 \\
Breast & 4 \\
Prostate & 2 \\
Uterus & 1 \\
Rectum & 1 \\
Colon & 1 \\
Bowel unspecified & 1 \\
Thyroid (anaplastic) & 1 \\
Multiple bladder neoplasia & 1 \\
Unspecified & 1 \\
Lymphosarcoma & 1 \\
Basal cell carcinoma of skin & 1 \\
Total & 20 \\
\hline
\end{tabular}

TABLE 5 Neoplasia in group treated with cytoxic drugs

\begin{tabular}{|c|c|c|}
\hline & Therapy & Duration (years) \\
\hline \multicolumn{3}{|l|}{ Carcinomas-deceased patients } \\
\hline Lung/bronchus & $\begin{array}{l}\text { Cyclophosphamide } \\
\text { Azathioprine }\end{array}$ & $\begin{array}{l}2 \cdot 3 \\
0 \cdot 3\end{array}$ \\
\hline Lung/bronchus & Azathioprine & 5 \\
\hline Lung/bronchus & $\begin{array}{l}\text { Chlorambucil } \\
\text { Azathioprine }\end{array}$ & $\begin{array}{l}1 \cdot 7 \\
3\end{array}$ \\
\hline Breast & $\begin{array}{l}\text { Intra-articular } \\
\text { cyclophosphamide }\end{array}$ & - \\
\hline Rectum & Azathioprine & 2 \\
\hline Unspecified & Azathioprine & $1 \cdot 5$ \\
\hline \multicolumn{3}{|l|}{ Carcinomas-prevalent } \\
\hline Uterus & Azathioprine & 8 \\
\hline Thyroid & Azathioprine & 3 \\
\hline Multiple bladder neoplasm & Cyclophosphamide & 8 \\
\hline Basal cell carcinoma & Azathioprine & 5 \\
\hline
\end{tabular}

The principal causes of death are listed in table 6 . Ischaemic heart disease heads the list, followed by neoplasia. Deaths from infections all involved the lung and the incidence is lower than previously recorded in patients with rheumatoid arthritis. ${ }^{24}{ }_{167}$

The observed death rate among the rheumatoid arthritis patients, from all causes according to age, has been compared with expected values obtained from the Registrar General's records using a $\chi^{2}$ test. The death rate was higher than expected in the 45 to 64-year-old age group, but lower in those aged 75 years or over. The differences are significant $(p<0.0005)$. Analysis of age related specific causes of death (table 7) showed an increased frequency of deaths from neoplasia and ischaemic heart disease occurring in the 45 to 64 -year-old age group, but this is not significant.

There was increased evidence of neoplasia in the patients with rheumatoid arthritis (table 3 ) but this was not associated with immunosuppressive treatment, nor was there any difference in duration of treatment between those developing tumours compared with the rest.

One patient developed a transitional cell carcinoma of the bladder after eight years' therapy with cyclophosphamide. This is of interest, as Wall and
TABLE 6 Causes of death in rheumatoid arthritis (46 patients)

\begin{tabular}{lrl}
\hline Cardiovascular & & \\
Ischaemic heart disease & 15 & $(7)^{*}$ \\
Cor pulmonale & 2 & $(1)$ \\
$\quad$ Pulmonary embolus & 2 & $(1)$ \\
Neoplasia & 13 & $(2)$ \\
Respiratory infections & 6 & $(3)$ \\
Chronic pulmonary disease & 3 & $(2)$ \\
Others & & $(2)$ \\
$\quad$ Adrenal failure & 2 & \\
Cirrhosis; portal hypertension & 1 & $(1)$ \\
Peptic ulceration & 1 & \\
Cervical subluxation & 1 & \\
\hline
\end{tabular}

${ }^{*}$ Number in parentheses indicates confirmed at necropsy.

TABLE 7 Rheumatoid arthritis: age related specific causes of death (1966-1976)

\begin{tabular}{|c|c|c|c|c|}
\hline \multirow{2}{*}{$\begin{array}{l}\text { Age } \\
\text { (yr) }\end{array}$} & \multicolumn{2}{|l|}{ Neoplasia } & \multicolumn{2}{|c|}{ Ischaemic heart disease } \\
\hline & Observed & Expected & Observed & Expected \\
\hline $25-44$ & - & $0 \cdot 1$ & - & 0.320 \\
\hline $45-64$ & 6 & $3 \cdot 3$ & 10 & $2 \cdot 626$ \\
\hline $65-74$ & 5 & $4 \cdot 6$ & 5 & $5 \cdot 117$ \\
\hline $75-$ & 2 & $1 \cdot 2$ & - & $2 \cdot 521$ \\
\hline Totals & 13 & $9 \cdot 4$ & 15 & $10 \cdot 584$ \\
\hline
\end{tabular}

Clausen in $1975^{232}$ described five cases of carcinoma of the bladder after prolonged cyclophosphamide therapy. In each case interstitial haemorrhagic cystitis preceded the development of the carcinoma. The patients all developed fatal and invasive carcinomas. Dale and Smith in $1974^{39}$ suggested that the tumour could be small and possibly non-recurrent. The patient in this study has had local recurrences which have been removed at routine cystoscopy.

The presence of haemorrhagic cystitis in a patient receiving cyclophosphamide should suggest immediate and permanent discontinuation of the drug. However, the cessation of haematuria and withdrawal of the drug does not eliminate the need for cystoscopy, and probably this should be repeated periodically, indefinitely thereafter. Previous observations have shown atypical epithelial cells on cytological examination of urine in haemorrhagic cystitis during treatment with cyclophosphamide. ${ }^{68} \mathrm{~A}$ necropsy study showed that ten of 49 patients who had received long-term cyclophosphamide had signs of urinary bladder fibrosis, the frequency being related to dose and duration of therapy. In about $50 \%$ of cases the fibrosis did not produce symptoms.

These findings suggest caution in the use of cyclophosphamide for non-malignant conditions. It is suggested that protracted periods of treatment should be avoided, and that the drug be used in intermittent courses if at all possible.

Louie and Schwart $\mathrm{z}^{130}$ compiled a list of 109 treated patients who developed neoplasms and compared them with patients who did not receive 
immunosuppressive therapy. The most prominent difference between the treated and untreated patients was the increased incidence of acute myeloblastic leukaemia in the treated group. The most important association was therapy with an alkylating agent. Certainly this review of the literature suggests that these agents are more likely to produce problems. However, assessment of the true risk is difficult as we do not know the expected number of neoplasms in untreated patients.

The potential oncogenic effect of immunosuppressive drugs remains one of the main deterrents to their wide-spread use in the treatment of rheumatoid arthritis. Further studies are required to assess the true influence of these drugs on the incidence of tumours in rheumatoid arthritis. 